\title{
CONCURRENT MULTI-SCALE MODELING OF COMPOSITES UNDER HIGH RATES OF LOADING
}

\author{
Michael May ${ }^{1}$, Stefan Hiermaier ${ }^{1}$ \\ ${ }^{1}$ Fraunhofer Institute for High-Speed Dynamics, Ernst-Mach-Institut, EMI, Eckerstraße 4, \\ 79104 Freiburg, Germany (michael.may@emi.fraunhofer.de)
}

\begin{abstract}
A computationally efficient concurrent multi-scale methodology for modeling composites under high rates of loading is proposed. The physically based model relies on micromechanical properties of the constituents only. Initially, the material response is calculated based on homogenized linear-elastic, orthotropic material properties at the laminate scale which are calculated using the rule of mixtures. A modified Hashin-Rotem criterion is then used to identify the switch to a more accurate micromechanical analysis based on the generalized method of cells (GMC). The methodology is verified using one-element tests on UDcomposites and validated against high-velocity impact experiments. Both, the correlation with the experiments and the computational efficiency are good.
\end{abstract}

Keywords: Composites, Multi-scale modeling, Impact.

\section{INTRODUCTION}

Several high-tech applications require the use of modern materials allowing tailoring the properties, especially stiffness, strength and crashworthiness, towards the respective loading conditions. Here, composite materials, due to their flexibility in design, offer large potential for application in structures subjected to complex loading. Currently there are no predictive engineering tools for designing safety critical composite structures against crash and impact loading. For a large scale industrial application of composites it is therefore inevitable to develop reliable design strategies and predictive simulation tools considering the special requirements for high rates of loading.

Predictive simulation of damage and failure in composite structures requires a physical foundation of the model as well as a high degree of computational efficiency At present, fulfilling both requirements at the macro-scale is a difficult task, as the non-linear processes occurring at different length scales, their evolution and interaction cannot be captured accurately using macroscopic properties. On the other hand, highly accurate micromechanical simulations, capturing these effects, are unfeasible due to the high computational expenses.

In this paper we try to address these issues by proposing a concurrent multi-scale methodology capable of switching between scales when necessary. 


\section{MODEL DESCRIPTION}

The methodology proposed here requires knowledge about the properties of the constituents (fiber and matrix) at the micro-scale. The backbone of this methodology is an extension $[2,8]$ to the method of cells originally proposed by Aboudi [1]. A computationally efficient implementation of this method was realized in EMI's in-house Hydro-code SOPHIA [8] following the suggestions by Pindera and Bednarczyk [11]. This formulation is briefly described in section 2.1 of this paper.

Although the formulation proposed by Pindera and Bednarczyk [11] is more efficient than the original method, the performance can still be improved by using a less sophisticated model during linear-elastic loading when the microstructural effects are not yet driving the material response. Therefore simulations are initially performed using the homogenized orthotropic linear-elastic material model, described in section 2.2.

This model is perfectly valid during elastic loading of the composite and becomes inaccurate once plasticity and damage initiate. Then a more sophisticated model is required. The criteria for switching between the two models are explained in section 2.3.

\subsection{Generalized method of cells}

The analytical multi-scale method of the "Generalized Method of Cells" allows performing physically based FE simulations at two length scales. During a finite element simulation at macroscopic length scale the microstructure of a composite is introduced at each integration point by means of a virtual representative volume element (RVE). The original MOC [1] relies on a subdivision of the representative volume element into four orthogonal cells, one cell for the fiber, three cells for the surrounding matrix as shown on the left hand side of Fig. 1. The subdivision into a higher number of orthogonal cells as shown on the right hand side of Fig. 1 was proposed in $[2,9]$ and allows a more accurate representation of the fiber geometry. Additionally, this enables the user to assess a wide variety of fiber distributions and their respective interactions as each cell can be assigned different properties.

Stipulating continuity conditions of forces and displacements between neighboring cells, the true normal and shear stress components in each cell can be calculated as follows.

$$
\sigma^{<\alpha \beta>}=\left[\begin{array}{c}
\sigma_{11}^{<\alpha \beta>} \\
T_{2}^{<\alpha>} \\
T_{3}^{<\beta>} \\
\sigma_{23}^{<\alpha \beta} \\
T_{13}^{<\beta>} \\
T_{12}^{<\alpha>}
\end{array}\right] .
$$




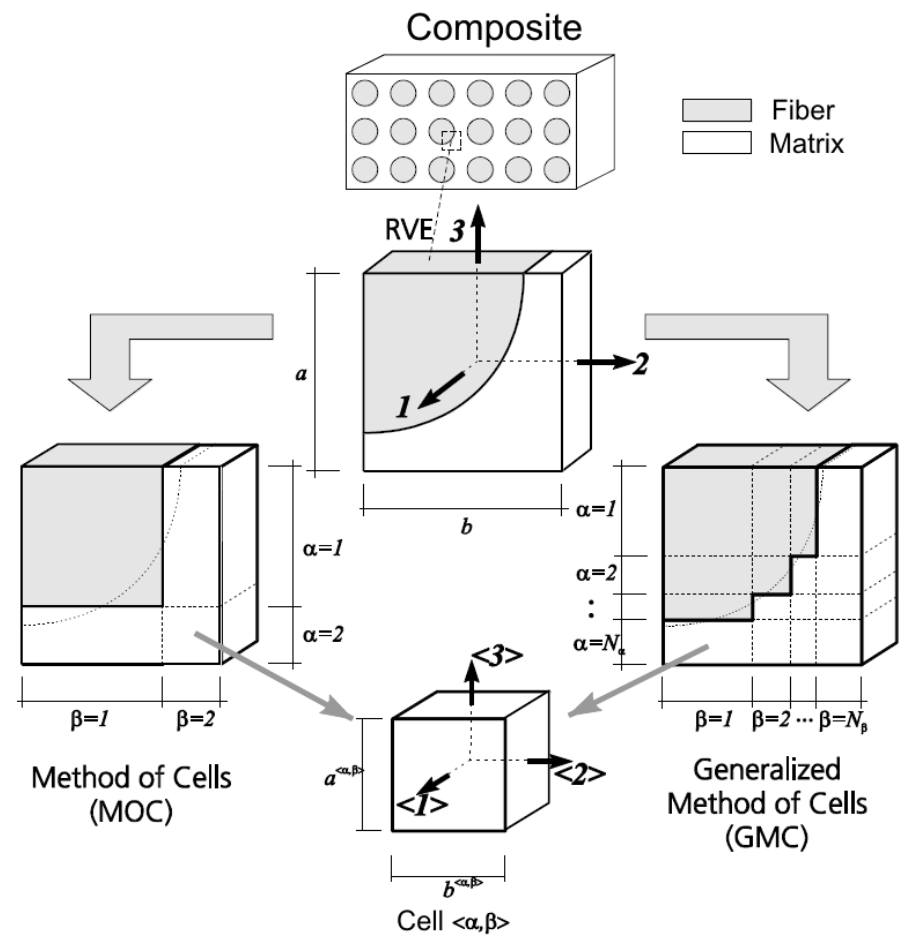

Figure 1. Principle of GMC [8].

The true normal stresses are given by

$$
\begin{gathered}
\sigma_{11}^{\langle\alpha \beta\rangle}=\frac{1-d^{\langle\alpha \beta\rangle}}{S_{11}^{\langle\alpha \beta}}\left(\varepsilon_{11}-\varepsilon_{p l .11}^{\langle\alpha \beta\rangle}-\frac{S_{12}^{\langle\alpha \beta\rangle}}{1-d^{\langle\alpha \beta\rangle}} \sigma_{22}^{\langle\alpha \beta\rangle}-\frac{S_{13}^{\langle\alpha \beta\rangle}}{1-d^{\langle\alpha \beta\rangle}} \sigma_{33}^{\langle\alpha \beta\rangle}\right) . \\
\left.\left\{\begin{array}{l}
T_{2}^{\langle\alpha\rangle} \\
T_{3}^{\langle\beta\rangle}
\end{array}\right\}=\left[\begin{array}{ll}
m_{22} & m_{23} \\
m_{32} & m_{33}
\end{array}\right]^{-1} \cdot\left[\begin{array}{l}
b \\
0
\end{array}\right\} \varepsilon_{22}+\left\{\begin{array}{l}
0 \\
a
\end{array}\right\} \varepsilon_{33}-\left\{\begin{array}{l}
c \\
e
\end{array}\right\} \varepsilon_{11}-\left\{\begin{array}{l}
p_{2} \\
p_{3}
\end{array}\right\}\right] .
\end{gathered}
$$

Here $a, b, c, e$ are submatrices containing information depending on the dimensions of the subcells, the vectors $p_{2}$ and $p_{3}$ contain information on plastic material properties, $m_{i j}$ are submatrices defined in [8,10], $S_{i j}$ are the components of the compliance tensor, $d^{<\alpha \beta>}$ is the cell damage, $\varepsilon_{i i}$ are the macroscopic normal true strains in i-direction, and $\sigma_{i i}{ }^{<\beta>}$ are the true cell normal stresses in i-direction.

The true shear stresses can be calculated as follows

$$
\sigma_{23}^{\langle\alpha \beta\rangle}=\frac{2 a b \varepsilon_{23}-2 \sum_{\alpha}^{N_{\alpha}} \sum_{\beta}^{N_{\beta}} a^{\langle\alpha \beta\rangle} b^{\langle\alpha \beta\rangle} \varepsilon_{p l .23}^{\langle\alpha \beta\rangle}}{\sum_{\alpha}^{N_{\alpha}} \sum_{\beta}^{N_{\beta}} a^{\langle\alpha \beta\rangle} b^{\langle\alpha \beta\rangle} \frac{S_{44}^{\langle\alpha \beta\rangle}}{1-d^{\langle\alpha \beta\rangle}}} .
$$




$$
\begin{gathered}
T_{12}^{\langle\alpha\rangle}=\frac{2 b \varepsilon_{12}-2 \sum_{\beta}^{N_{\beta}} b^{\langle\alpha \beta\rangle} \varepsilon_{p l .12}^{\langle\alpha \beta\rangle}}{\sum_{\beta}^{N_{\beta}} b^{\langle\alpha \beta\rangle} \frac{S_{66}^{\langle\alpha \beta\rangle}}{1-d^{\langle\alpha \beta\rangle}}} . \\
T_{13}^{\langle\beta\rangle}=\frac{2 a \varepsilon_{13}-2 \sum_{\alpha}^{N_{\alpha}} a^{\langle\alpha \beta\rangle} \varepsilon_{p l .13}^{\langle\alpha \beta\rangle}}{\sum_{\alpha}^{N_{\alpha}} a^{\langle\alpha \beta\rangle} \frac{S_{55}^{\langle\alpha \beta\rangle}}{1-d^{\langle\alpha \beta\rangle}}} .
\end{gathered}
$$

The macroscopic stress and strain response follow from homogenization [9].

$$
\begin{aligned}
& \sigma=\frac{1}{a b} \sum_{\alpha=1}^{N_{\alpha}} \sum_{\beta=1}^{N_{\beta}} a^{<\alpha, \beta>} b^{<\alpha, \beta>} \tilde{\sigma}^{<\alpha, \beta>} . \\
& \varepsilon=\frac{1}{a b} \sum_{\alpha=1}^{N_{\alpha}} \sum_{\beta=1}^{N_{\beta}} a^{<\alpha, \beta>} b^{<\alpha, \beta>} \widetilde{\mathcal{E}}^{<\alpha, \beta>} .
\end{aligned}
$$

For further information on the derivation of the equations listed above and the implementation of the GMC into a FE software, the interested reader is referred to work by Nossek [8] or Pindera and Bednarcyk [11].

\subsection{Homogenized orthotropic linear-elastic response}

Homogenized orthotropic linear-elastic properties follow from the rule of mixtures. The homogenized elastic Young's modulus in fiber direction is then calculated as follows:

$$
E_{11}=E_{1 f} \cdot V_{f}+E_{m} \cdot\left(1-V_{f}\right)
$$

Here, $E_{l f}$ is the axial Young's modulus of the fiber, $E_{m}$ is the Young's modulus of the resin, and $V_{f}$ is the fiber volume fraction.

Assuming transverse isotropy, the transverse moduli follow from

$$
E_{22}=E_{33}=\frac{E_{2 f} \cdot \frac{E_{m}}{1-v_{m}^{2}}}{\frac{E_{m}}{1-v_{m}^{2}} \cdot V_{f}+E_{2 f} \cdot\left(1-V_{f}\right)} .
$$

Here, $E_{2 f}$ is the transverse Young's modulus, $E_{m}$ is the Young's modulus of the resin, and $V_{f}$ 
is the fiber volume fraction.

The homogenized Poisson's ratio follows from

$$
v_{12}=v_{13}=v_{12 f} \cdot V_{f}+v_{m} \cdot\left(1-V_{f}\right) \text {. }
$$

$v_{12 f}$ and $v_{m}$ are the Poisson's rations of the fiber and the matrix, respectively.

For a first approximation the out-of-plane Poisson's ratio $v_{23}$ can be estimated using the inplane Poisson's ratio

$$
v_{23} \approx v_{12} \text {. }
$$

Still assuming transverse isotropy, the homogenized in-plane shear moduli are then calculated as follows [3]:

$$
G_{12}=G_{13}=\frac{G_{12 f} \cdot \frac{E_{m}}{2 \cdot\left(1+v_{m}\right)}}{G_{12 f} \cdot\left(1-V_{f}\right)+\frac{E_{m}}{2 \cdot\left(1+v_{m}\right)} \cdot V_{f}} .
$$

Hashin [4] showed that the out-of plane shear modulus can be approximated using the relationship

$$
G_{23}=\frac{E_{2}}{2 \cdot\left(1+v_{23}\right)} .
$$

With all important material parameters known, the orthotropic stiffness matrix can be completed. In order to reduce the computational effort this is only done in the first time step, stored and recalled in succeeding time steps.

\subsection{Criterion for change of scale}

Having two independent methods in place, the next challenge is to provide a link between the two methods. Therefore a criterion has to be found allowing identifying which material model provides the best compromise of accuracy and computational efficiency for a particular element under a certain stress state. As the orthotropic model described in the previous section is only valid during the initial linear-elastic loading phase a criterion must be able to detect the onset of damage and plasticity. Hashin and Rotem [4] suggested very simple failure criteria for composites which make a distinction of cases for fiber failure and matrix dominated failure as shown below 


$$
\begin{gathered}
\frac{\left|\sigma_{1}\right|}{F_{1}}=1 . \\
\left(\frac{\sigma_{2}}{F_{2}}\right)^{2}+\left(\frac{\tau_{4}}{F_{4}}\right)^{2}+\left(\frac{\tau_{6}}{F_{6}}\right)^{2}=1 \\
\left(\frac{\sigma_{3}}{F_{3}}\right)^{2}+\left(\frac{\tau_{4}}{F_{4}}\right)^{2}+\left(\frac{\tau_{5}}{F_{5}}\right)^{2}=1 .
\end{gathered}
$$

Due to the simplicity of this criterion, which again provides computational advantages towards more sophisticated models such as a Puck failure criterion [10], we chose to adapt a modified Hashin-Rotem criterion as an indicator for the necessity to change scales. The fiber failure criterion was rewritten as follows

$$
\frac{\left|\sigma_{1}\right|}{V_{f} \cdot \sigma_{1 f}}=\chi_{1} .
$$

Here, $\chi_{1}$ is a parameter in the range of $[0,1]$. For a value of 0 the formulation would directly call the routine for the GMC; for a value of 1 the GMC would be called at fiber failure. As loading in fiber direction, at least for carbon composite materials, is mainly linear elastic, switching between the homogenized model and the accurate model can be done at a late loading stage, e.g. $\chi_{1}=0.9$.

This is different for the matrix dominated failure modes where non-linear behavior occurs very early in the loading history. Here, the Hashin-Rotem criterion was reformulated as follows

$$
\begin{aligned}
& \left(\frac{\sigma_{2}}{\chi_{2} \cdot \sigma_{m}^{0}}\right)^{2}+\left(\frac{\tau_{4}}{\chi_{2} \cdot \sigma_{m}^{0}}\right)^{2}+\left(\frac{\tau_{6}}{\chi_{2} \cdot \sigma_{m}^{0}}\right)^{2}=1 . \\
& \left(\frac{\sigma_{3}}{\chi_{2} \cdot \sigma_{m}^{0}}\right)^{2}+\left(\frac{\tau_{4}}{\chi_{2} \cdot \sigma_{m}^{0}}\right)^{2}+\left(\frac{\tau_{5}}{\chi_{2} \cdot \sigma_{m}^{0}}\right)^{2}=1 .
\end{aligned}
$$

The parameter $\chi_{2}$ was introduced to scale-down the yield stress $\sigma_{m}^{0}$ of pure resin which was taken as a reference for changeing scale. Again, $\chi_{2}$ is in the range of $[0,1]$, however as nonlinearities may start at very low load levels, chosing the parameter $\chi_{2}$ in the range of $[0.3,0.7]$ may be advantageous in order to avoid spurious oscillations occuring during switch from the approximate orthotropic linear-elastic stress-strain response to the accurate multiscale stress-strain response. 


\subsection{Strain rate dependent material behavior}

The main focus of this paper is on carbon/epoxy composites. As carbon fibers are not known to exhibit rate dependent behavior, the same material response for the fiber was assumed for all loading rates. However, epoxy resins may respond sensitive to dynamic loading. In this paper, following Nossek [8], rate sensitivity is introduced using a logarithmic relationship for the yield stress

$$
\sigma_{m}^{y}=\sigma_{m}^{0}+m \cdot \log \frac{\dot{\varepsilon}_{v}}{\dot{\varepsilon}_{v, 0}}
$$

Here, $\sigma_{m}^{y}$ is the rate dependent yield stress of the resin, $\sigma_{m}^{0}$ is the quasistatic reference yield stress of the resin, $m$ is a coefficient describing the slope of the curve, $\dot{\varepsilon}_{v}$ is the equivalent strain rate, and $\dot{\varepsilon}_{v 0}$ is the equivalent reference strain rate at quasi-static loading.

\section{MODEL VERIFICATION}

The model was implemented into our in-house code SOPHIA. The model was calibrated with micro-mechanical data for Torayca Carbon Fibers, type T700GC-12000-31E400KA8A, and resin Hexion EPR L20 + EPH 960. The required rate-dependent micromechanical data was taken from Nossek [8]. The model was then verified using singleelement models loaded in axial and transverse tension. The numerical results are shown in the sections below.

\subsection{Axial loading}

A single-element model with UD fiber orientation and $40 \%$ fiber volume fraction was loaded in fiber direction. As suggested in section 2.3, $\chi_{1}$ was set to 0.9. For axial loading $\chi_{2}$ does not influence the predictions as only fiber failure is triggered, so the value was set to 0.9 as well. Figure 2 compares the predictions obtained by the new formulation combining the models described in sections 2.1 and 2.2 with the accurate solution given by the GMC. It can be seen that the transition between the two different material models works smoothly and the overal solution remains unchanged. 


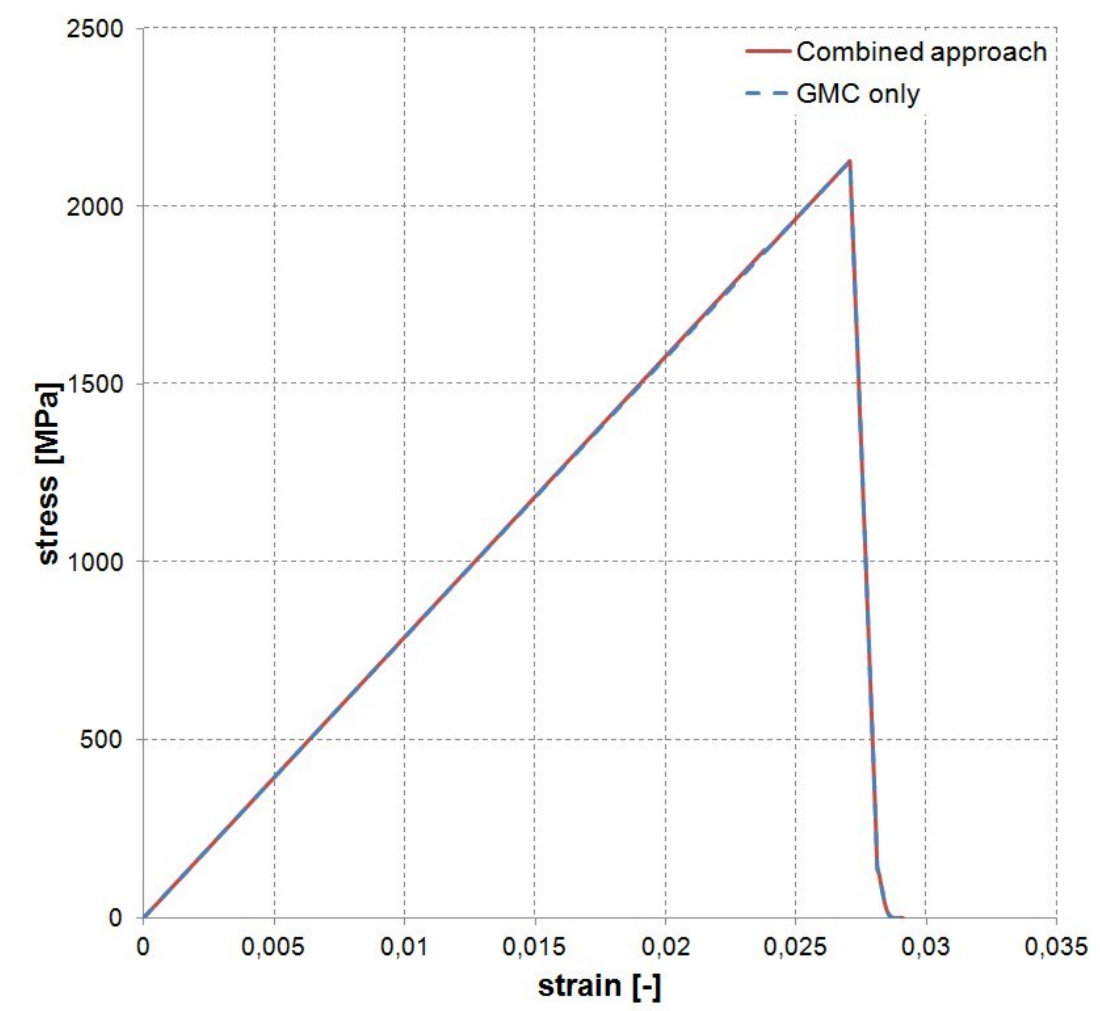

Figure 2. Comparison of numerical predictions for axial loading.

\subsection{Transverse loading}

In a second step, the single-element model with UD fiber orientation and $40 \%$ fiber volume fraction was loaded in transverse direction. $\chi_{1}$ was still set to 0.9 . For transverse loading $\chi_{2}$ becomes more important. Different values of $\chi_{2}$ were tested in order to assess the effect of changing this parameter. Figure 3 compares the predictions for different values of $\chi_{2}$ with the predictions by the GMC. It can be seen that spurious oscillations occur at the moment the formulation is changed. This is due to two reasons. Firstly, for the calculation of transverse properties in the homogenized model a hexagonal fiber distribution is assumed whilst for this particular simulation, the GMC was fed with a square fiber distribution. Secondly, for this particular resin non-linearities start at very low levels of stress leading to deviations from the linear-elastic formulation. Smaller values of $\chi_{2}$ lead to predictions with less spurious oscillations caused by the change of model as the difference in stress and therefore the stress jump is smaller if the model is changed during early stages of the simulation. The drawback of this is that the gain in computational performance is limited by the desired cleanliness of the stress-strain curve. 


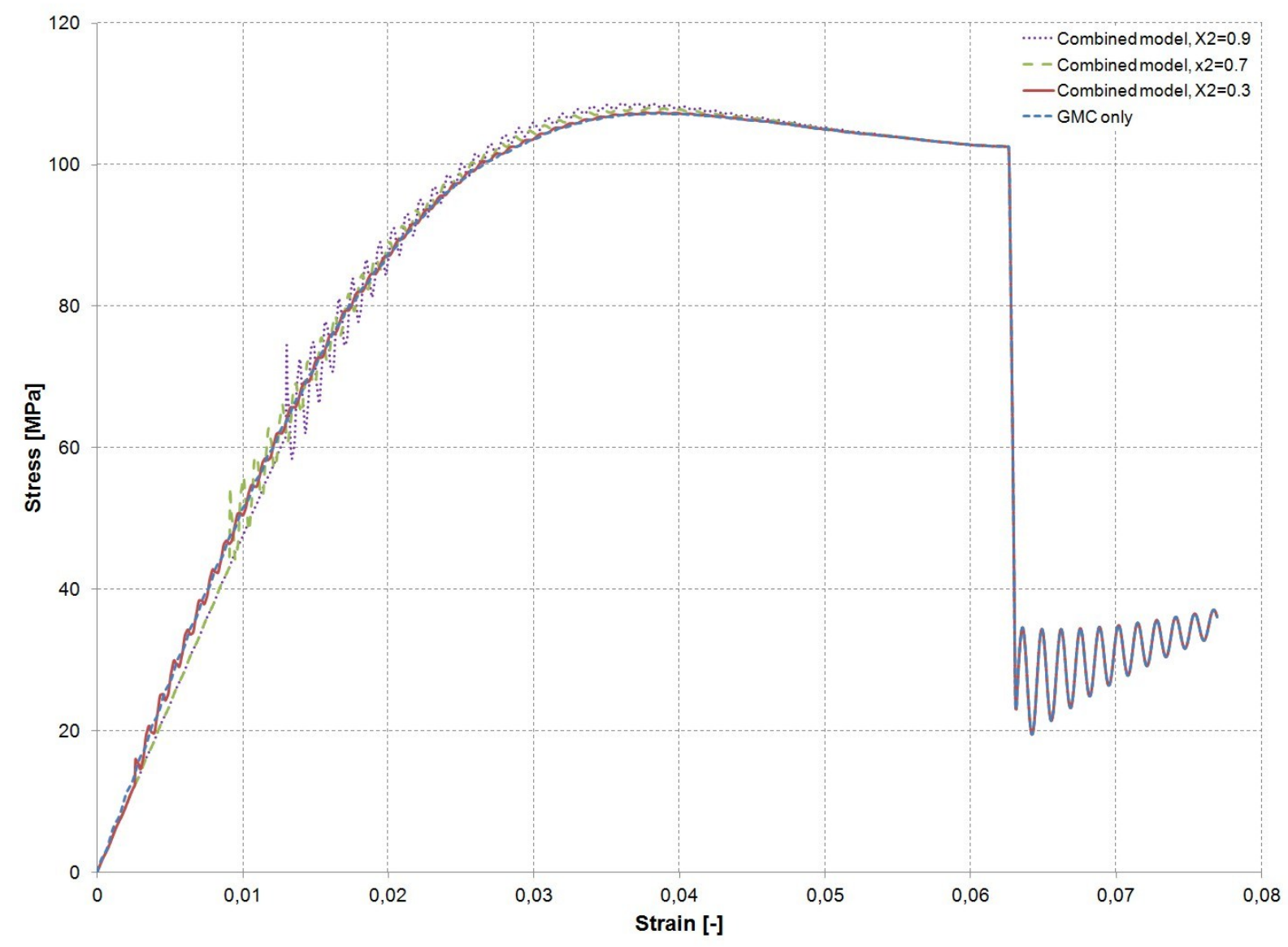

Figure 3. Comparison of numerical predictions for transverse loading.

\section{MODEL VALIDATION}

The model is validated by simulating high velocity impact of a steel sphere onto a carbon composite plate of size $200 \times 220 \mathrm{~mm}^{2}$, layup $\left[(0 / 45 / 90 /-45)_{2}\right]_{\mathrm{s}}$ and fiber volume fraction $\mathrm{V}_{\mathrm{f}}=59 \%$. The experiments are quickly described in section 4.1. Details can be found in the work of Nossek [8]. The setup of the FE model and the simulation results are then described in section 4.2 .

\subsection{Experiments}

High velocity impact tests were performed at EMI using the experimental setup shown in Fig. 4. Steel spheres of diameter $7 \mathrm{~mm}$ were accelerated to velocities in the range of 230 $\mathrm{m} / \mathrm{s}$ to $550 \mathrm{~m} / \mathrm{s}$. The targets were quasi-isotropic composite plates of layup $\left[(0 / 45 / 90 /-45)_{2}\right]_{\mathrm{s}}$. The composite was a carbon/epoxy made from Torayca Carbon Fibers, type T700GC-1200031E-400KA8A, and resin Hexion EPR L20 + EPH 960. The plates were mounted in a rigid steel frame leaving a target size of $200 \times 200 \mathrm{~mm}$. The velocity of the projectile was measured just before and directly behind the target using light barriers and high speed cameras. Post 
mortem C-scan analyses were performed in order to evaluate the extent of delamination damage in the composite plate.

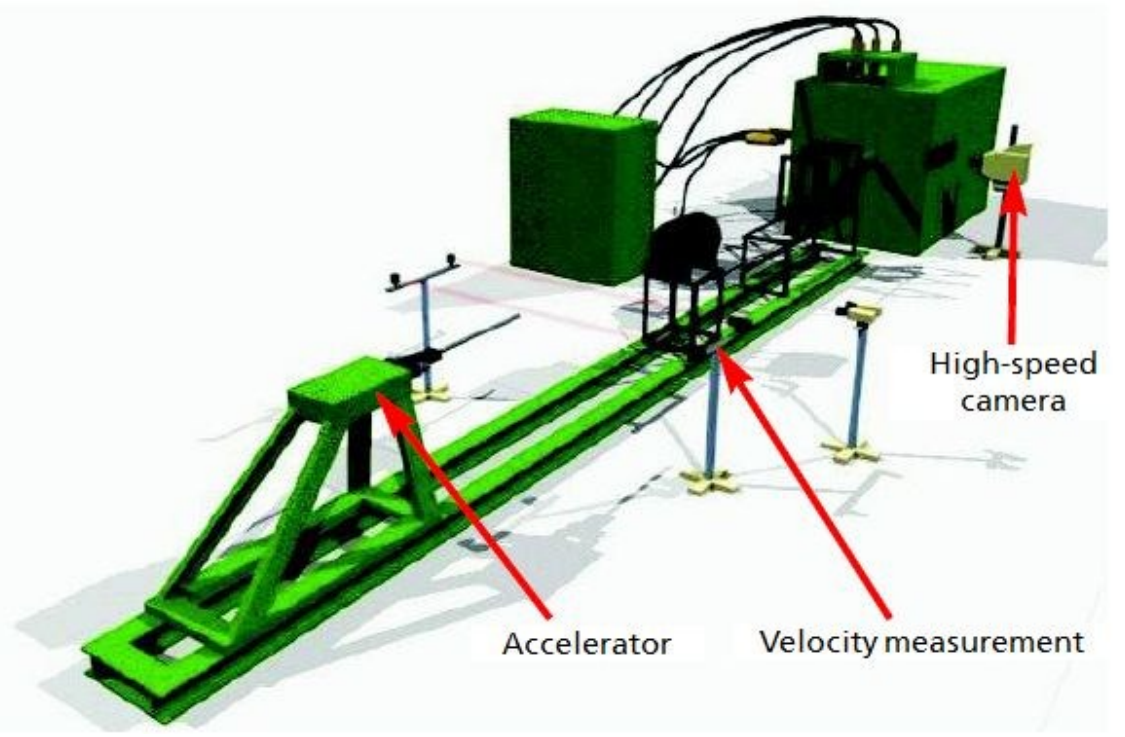

Figure 4. Experimental setup.

A Lambert-Jonas curve [6,7] was approximated predicting the ballistic limit of $v_{b l}=233 \mathrm{~m} / \mathrm{s}$. It was found that the extent of delamination decreased with increasing impact velocity.

\subsection{Simulation}

The finite element model used for validation is shown in Fig. 5. A total of 84,768 eight-noded, fully integrated solid elements were used for discretization of the composite plate. Pressure averaging was used in order to avoid element locking. The element size chosen was $2 \mathrm{~mm}$. The mesh was refined to $0.75 \mathrm{~mm}$ in the vicinity around the impact. The steel sphere was modeled with a total of 1,824 solid elements with linear-elastic material properties. Interlaminar behavior of the composite plate was modeled using initially rigid cohesive elements as suggested in [8]. Two different types of constitutive models were used for modeling the intralaminar behavior of the UD plies (GMC only and the proposed combined GMC + homogenized model). This allows a direct comparison of the accuracy and computational efficiency of the GMC approach suggested in [8] and the novel formulation proposed in this paper. It should be noted that Nossek [8] compared several variations of the GMC in terms of their accuracy and computational efficiency. Only the formulation with the best performance in terms of accuracy and computational efficiency is used here.

Figure 6 compares the predicted damage in the central region for an impact velocity of $400 \mathrm{~m} / \mathrm{s}$. It can be seen that both simulations correctly predict a full penetration of the projectile. Also, the location and extent of delamination (green) is comparable. The simulation time for the combined model using switching parameters $\chi_{1}=0.9$ and $\chi_{2}=0.7$ was about $50 \%$ of the simulation time for a GMC only model. 

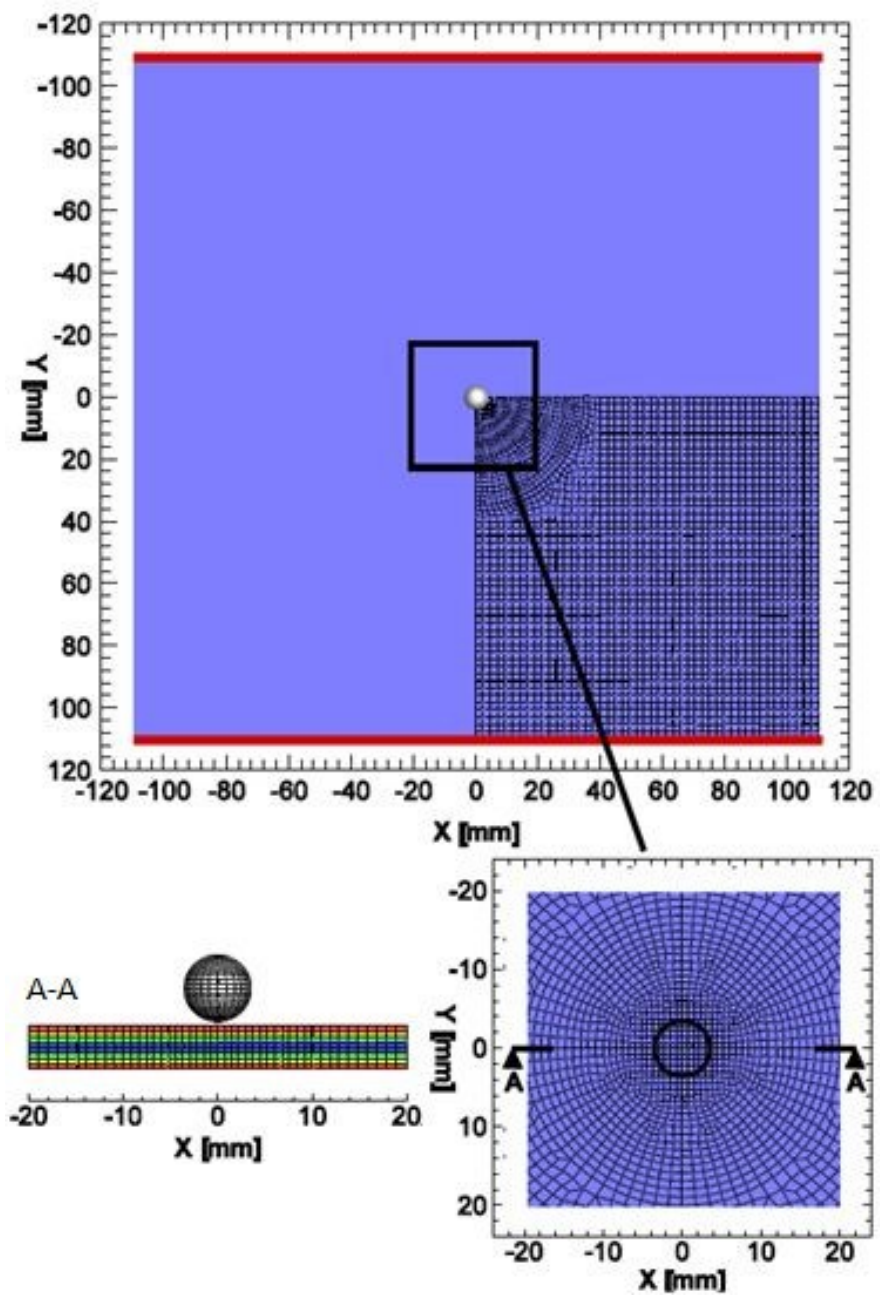

Figure 5. Model setup.

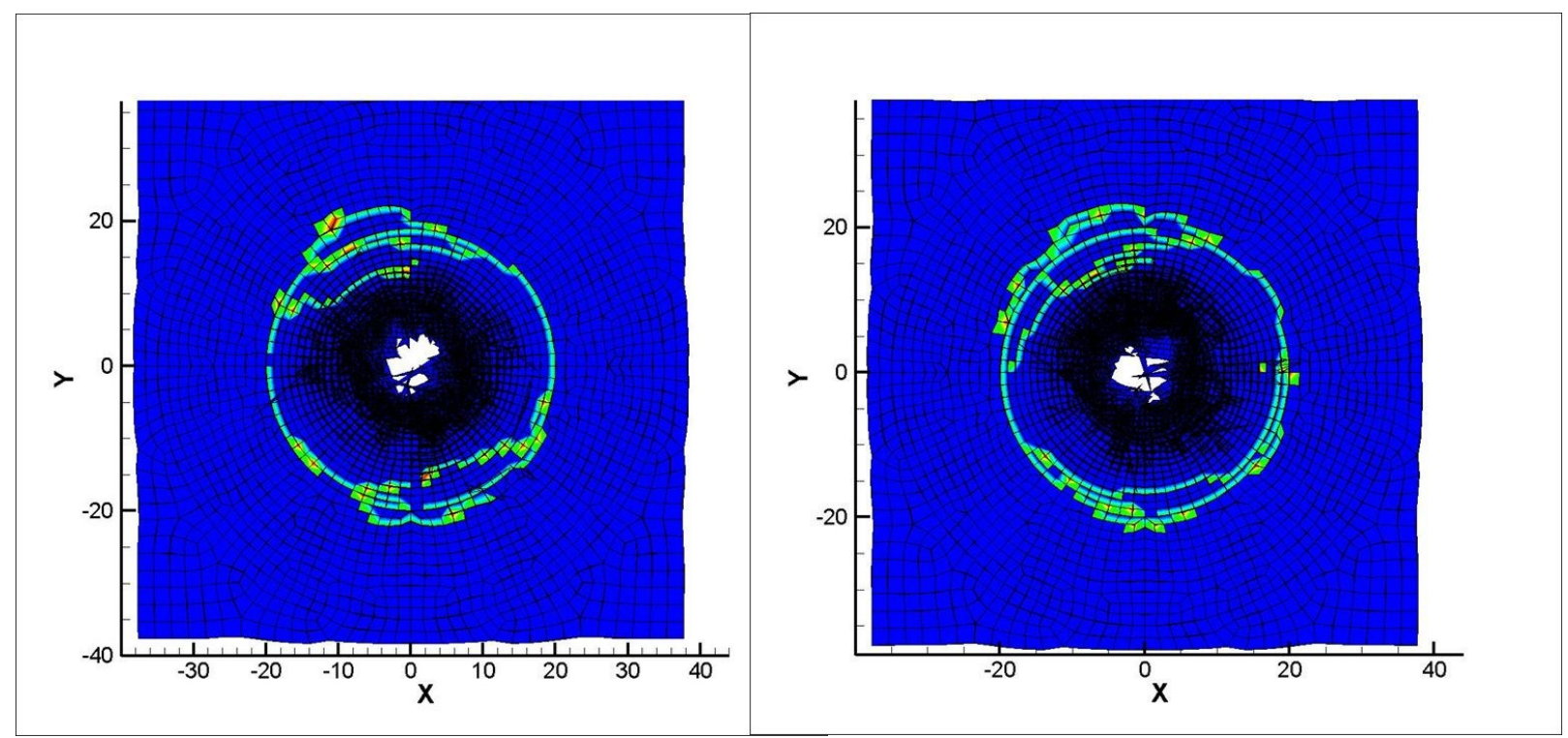

Figure 6 . Predicted damage for an impact velocity of $400 \mathrm{~m} / \mathrm{s}$ for a GMC only simulation (left) and a combined simulation (right). 
Simulations were also performed for an impact velocity of $230 \mathrm{~m} / \mathrm{s}$ which is just under the ballistic limit. The expected response is therefore that the composite plate is not fully penetrated by the projectile. This is also captured by the simulations using switching parameters $\chi_{1}=0.9$ and $\chi_{2}=0.3$. The relatively small value for the parameter $\chi_{2}$ was chosen as, unlike for the faster impact velocity, using $\chi_{2}=0.7$ lead to wrong results (perforation of the composite target). This was attributed to the spurious oscillations causing premature damage and failure. Due to this low value of $\chi_{2}$, the performance gain for the proposed combined formulation was reduced to about $30 \%$ compared against the GMC only simulations. Figure 7 shows a section cut through the target and a top view on target at $t=250 \mathrm{~ms}$, just before the projectile comes to a complete arrest.

For all cases under investigation the predicted delamination size was larger than the experimentally measured delamination size. However, as this phenomenon occurred with both formulations, this was not caused by the simplifications underlying the proposed concurrent multi-scale methodology. Using a rate dependent cohesive zone formulation could improve the prediction of the delamination size.
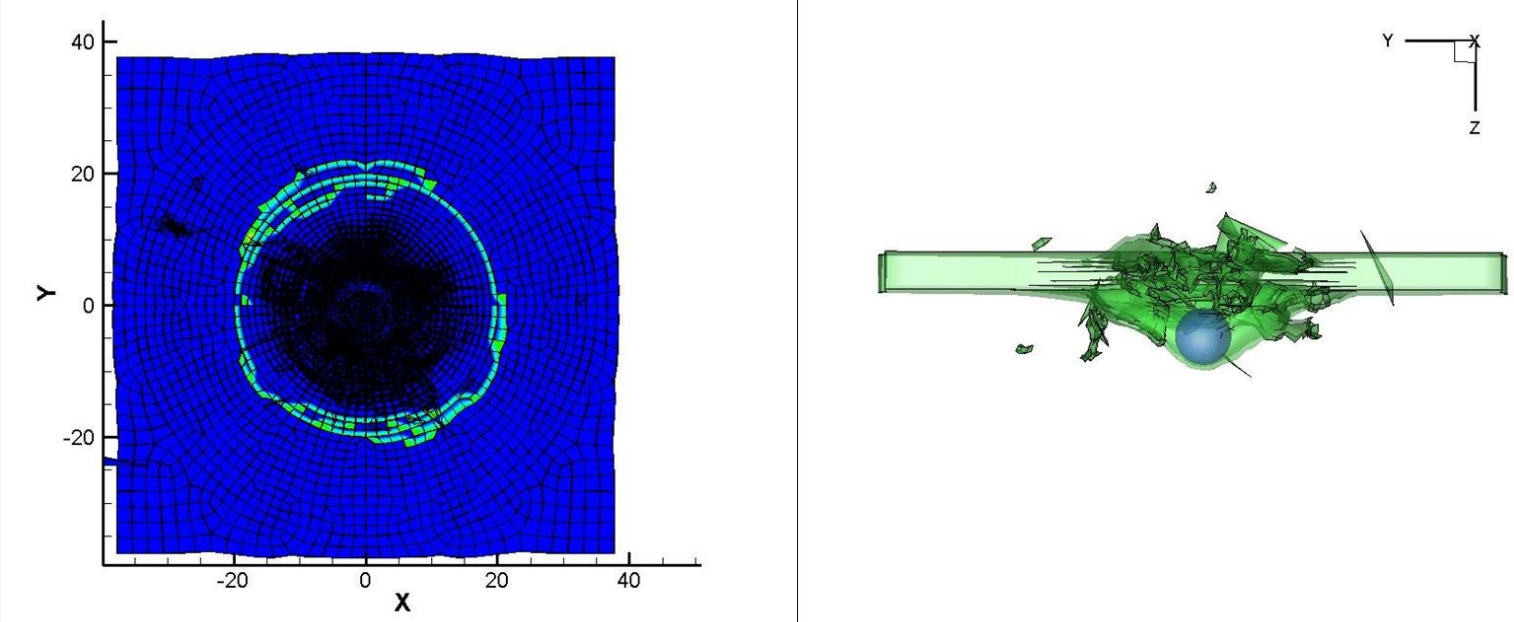

Figure 7. Predicted damage for an impact velocity of $230 \mathrm{~m} / \mathrm{s}$ for a combined simulation. The top view is shown on the left hand side, a section cut through the target showing the projectile which has come to arrest is shown at the right hand side.

\section{CONCLUSIONS}

A novel, computational efficient, concurrent multi-scale methodology is proposed for modeling impact on composite structures. Initially, the material is described using homogenized linear-elastic, orthotropic material properties obtained by applying the rule of mixtures to the properties of the constituents. A modified Hashin-Rotem criterion is used to assess the validity of this material description. Once the simple description is not valid anymore, the more accurate but also computationally more expensive analytical multi-scale approach 
"Generalized Method of Cells" is activated. The proposed methodology was successfully verified using single-element tests. Then the formulation was applied to the complex case of high velocity impact on a composite plate. The accuracy of the prediction was comparable to simulations performed with the GMC only. At the same time the simulation time for a high velocity impact model was reduced significantly (up to $50 \%$ ). The proposed methodology has therefore shown to be a useful tool for modeling high velocity impact on composites.

\section{Acknowledgements}

The authors would like to thank the Fraunhofer society for funding through the Attract programme IMAGINE.

\section{REFERENCES}

[1] Aboudi J., "Mechanics of Composite Materials - A Unified Micromechanical Approach", Elsevier, 1991.

[2] Aboudi J., "Micromechanical Analysis of Composites by the Method of Cells - Update", Appl. Mech. Rev. 49, 83-91, 1996.

[3] Daniel I. M., Ishai O., "Engineering Mechanics of Composite Materials - second edition", Oxford University Press, 2006.

[4] Hashin Z., Rotem A., "A fatigue Failure Criterion for Fiber Reinforced Materials", $J$. Compos. Materials 7, 448-464, 1973.

[5] Hashin Z., "Analysis of Composite Materials - A Survey", J. Appl. Mech. 50, 481-505, 1983.

[6] Lambert J. P., Jonas, G.H., "Towards Standardization of Terminal Ballistic Testing: Velocity Representation”, Report BRL-R-1852, Ballistic Res. Lab, Aberdeen, MD, 1976.

[7] Lambert J. P., "A Residual Velocity Predictive Model for Long Rod Penetrators", Report ARBRL-MR-02828, Ballistic Res. Lab, Aberdeen, MD, 1978.

[8] Nossek M., "Multiskalenmodllierung von Impaktbelastungen auf Faserverbundlaminate: Methodenentwicklung, Parameteridentifikation und Anwendung". $\dot{\varepsilon}-$ Forschungsergebnisse aus der Kurzzeitdynamik 18, Thoma K., Hiermaier S. (Eds.), ISBN 978-3-83960226-3, 2011.

[9] Paley M., Aboudi J., "Micromechanical Analysis of Composites by the Generalized Method of Cells Model", Mechanics of Materials 14, 127-139, 1992.

[10] Knops M., "Analysis of Failure in Fiber Polymer Laminates - The Theory of Alfred Puck", Springer, 2008.

[11] Pindera M.J., Bednarcyk, B.A., “An Efficient implementation of the Generalized Method of Cells for Unidirectional, Multi-Phased Composites with Complex Microstructures", Composites: Part B. 30, 87-105, 1999. 